УДК 517.53

\author{
M. V. Zabolotskyi, Yu. V. Basiuk, M. R. Mostova
}

\title{
BINOMIAL ASYMPTOTICS FOR THE LOGARITHMIC DERIVATIVE OF ZERO-ORDER ENTIRE FUNCTIONS WITH ZEROS ALONG CURVES OF REGULAR ROTATION
}

\begin{abstract}
M. V. Zabolotskyi, Yu. V. Basiuk, M. R. Mostova. Binomial asymptotics for the logarithmic derivative of zero-order entire functions with zeros along curves of regular rotation, Mat. Stud. 52 (2019), 156-165.

The relationship between the regular behavior of the logarithmic derivative of zero-order entire function $f$ with zeros on a finite system of curves of regular rotation for $f$ and the existence of $v$-density of zeros of $f$ along such curves is investigated.
\end{abstract}

1. Introduction. Let $H_{+}(\rho)$ be the class of entire functions $f$ with positive order $\rho, \rho(r)$ be a proximate order of $f, \rho(r) \rightarrow \rho$ as $r \rightarrow+\infty$. The creation by B. Levin and A. Pflüger of the theory of functions of completely regular growth (c.r.g.) for the class $H_{+}(\rho)$ became the main event of 20th century in the theory of analytic functions. In the case of noninteger $\rho$ the function $f \in H_{+}(\rho)$ is of c.r.g. if and only if zeros of $f$ have angular density with respect to the comparison function $V(r)=r^{\rho(r)}$.

Asymptotics and evaluations of the logarithmic derivative $F(z)=z f^{\prime}(z) / f(z)$ of entire functions $f$ outside exceptional sets play an important role in various fields of mathematics. Estimation of the modulus of the logarithmic derivative is important in the Nevanlinna theory and its applications to differential, functional and difference equations. Respectively necessary and sufficient conditions of affiliation $f \in H_{+}(\rho)$ to the set of functions of c.r.g. in terms of the logarithmic derivative are found in $[3]$ and $[5]<$ respectively.

In [1] there is generalized the theory of functions of c.r.g. in the case of the arrangement of zeros along curves of regular rotation (c.r.r.) and in particular along logarithmic spirals. We note that the asymptotics of canonical products with zeros lying on one ray or on c.r.r. is substantially used in [3] and [1], respectively.

From the results of [6] it follows that if we similarly introduce the notion of c.r.g. for the class $H_{0}$ of zero-order entire functions, then the obtained theory becomes ineffective. Indeed, c.r.g. of $f \in H_{0}$ is independent of the arguments of its zeros and depends only on their moduli; c.r.g. in $\mathbb{C}$ follows from c.r.g. on one ray; indicator of an entire function $f$ of c.r.g. from the class $H_{0}$ is a positive constant. The notion of strongly regular growth (s.r.g.) was introduced by the first author of this article in the 90th of the last century for the class $H_{0}$, which has features similar to the features of functions of c.r.g. from the class $H_{+}(\rho)$

2010 Mathematics Subject Classification: 30D15, 30D35.

Keywords: logarithmic derivative; entire function; order zero; binomial asymptotics; curves of regular rotation. doi:10.30970/ms.52.2.156-165

(C) M. V. Zabolotskyi, Yu. V. Basiuk, M. R. Mostova, 2019 
([8]). Criteria of s.r.g. for functions $f \in H_{0}$ in terms of quantities related to their logarithmic derivatives are found in $[10,11]$.

In this paper we consider the question of the connection between the regular behavior of the logarithmic derivative of a function $f$ of the class $H_{0}$, with zeros on c.r.r. and the existence of $v$-density of zeros of $f$ along such curves. We note that the Valiron-type and ValironTitchmarsh-type theorems on the relationship between the regular behavior of logarithm of $f$, which belongs to the class $H_{0}$, and the asymptotics of the counting function of zeros of $f$, which are located on a logarithmic spiral, are proved in [9].

2. Definitions and main results. By $L$ we denote the class of nonnegative continuously differentiable increasing to $+\infty$ on $\mathbb{R}_{+}$functions $v$ such that $\psi(r ; v):=r v^{\prime}(r) / v(r) \rightarrow 0$ as $r \rightarrow+\infty$. It is well known that accurate to equivalent functions the class $L$ coincides with the class of slowly increasing functions. Note also that functions $v$ of the class $L$ is of order zero, namely

$$
\varlimsup_{r \rightarrow+\infty} \ln ^{+} v(r) / \ln r=0 .
$$

We denote by $H_{0}(v)$ the class of entire functions of order zero, the counting function $n(r)=n(r, 0, f)$ of which zeros satisfies the condition $n(r)=O(v(r)), r \rightarrow+\infty$. For $\theta \in \mathbb{R}$, $1 \leq a<+\infty$ we set $l_{\theta}^{\gamma}(a, r)=\left\{z=t e^{i(\theta+\gamma(t))}: a \leq t \leq r\right\}, l_{\theta}^{\gamma}(1,+\infty)=l_{\theta}^{\gamma}$, where $\gamma(t)$ is a real-valued function defined on $[a, r]$.

Definition. We say that the curve $l_{\theta}^{\gamma}$ is called a curve of regular rotation (c.r.r.) for $f \in H_{0}(v)$ if $\gamma(t)$ is continuously differentiable on $[1,+\infty)$ function such that $(c \in \mathbb{R})$

$$
t \gamma^{\prime}(t)-c=o(\psi(t ; v)), t \rightarrow+\infty
$$

Let

$$
\Gamma_{m}^{\gamma}=\Gamma_{m}^{\gamma}(f)=\bigcup_{j=1}^{m} l_{\theta_{j}}^{\gamma},-\pi \leq \theta_{1}<\theta_{2}<\ldots<\theta_{m}<\pi,
$$

be a finite system of c.r.r. $l_{\theta_{j}}^{\gamma}$ for $f, h\left(\varphi ; \theta_{j}\right)=\varphi-\theta_{j}-\pi$ for $\varphi \in\left(\theta_{j}, \theta_{j}+2 \pi\right), \widehat{h}_{j}(\varphi)$ be the $2 \pi$-periodic continuation of the function $h\left(\varphi ; \theta_{j}\right)$ from $\left(\theta_{j}, \theta_{j}+2 \pi\right)$ to $\mathbb{R}$,

$$
h_{f}(\varphi)=\sum_{j=1}^{m} \Delta_{j} \widehat{h}_{j}(\varphi)
$$

for $\varphi \in[-\pi, \pi), \Delta_{j}>0, \varphi \neq \theta_{j}, n\left(r ; \theta_{j}\right)=n\left(r, 0, f ; \theta_{j}\right)$ be the number of zeros of $f$, which lie on $l_{\theta_{j}}^{\gamma}$, in the disk $\{z:|z| \leq r\}, \Delta=\Delta_{1}+\ldots+\Delta_{m}$. We denote by

$$
D^{\gamma}(r ; \alpha, \beta)=\bigcup_{\alpha \leq \varphi<\beta} l_{\varphi}^{\gamma}(1, r)
$$

the curvilinear sector, $-\pi \leq \alpha<\beta \leq \pi$.

We will say that zeros of $f \in H_{0}(v), v \in L$, have $v$-density $\Delta^{\gamma}(\alpha, \beta)$ along c.r.r. $l_{\varphi}^{\gamma}$ for $f$, if for all $\alpha, \beta \in[-\pi, \pi]$ except possibly a countable set the limit

$$
\lim _{r \rightarrow+\infty} \frac{n^{\gamma}(r ; \alpha, \beta)}{v(r)}=\Delta^{\gamma}(\alpha, \beta)
$$

exists, where $n^{\gamma}(r ; \alpha, \beta)$ is the number of zeros which lie in the curvilinear sector $D^{\gamma}(r ; \alpha, \beta)$. 
By $H_{0}\left(v, \Gamma_{m}^{\gamma}\right)$ we denote the subclass of functions of the class $H_{0}(v)$ with zeros on $\Gamma_{m}^{\gamma}$. For $\widetilde{v} \in L$ we set

$$
v(r)=\int_{1}^{r} \frac{\widetilde{v}(t)}{t} d t
$$

It is easy to see that $v \in L, \widetilde{v}(r)=o(v(r)), r \rightarrow+\infty$.

Theorem 1. Let $\widetilde{v} \in L, f \in H_{0}\left(v, \Gamma_{m}^{\gamma}\right), z=r e^{i(\varphi+\beta(r))}, \beta(r)-\gamma(r) \rightarrow 0$ as $r \rightarrow+\infty$. If for $j=\overline{1, m}$

$$
n\left(r ; \theta_{j}\right)=\Delta_{j} v(r)+o(\widetilde{v}(r)), r \rightarrow+\infty,
$$

then for $\varphi \in[-\pi, \pi), \varphi \neq \theta_{j}$,

$$
F(z)=\Delta v(r)+\frac{i}{1+i c} h_{f}(\varphi) \widetilde{v}(r)+o(\widetilde{v}(r)), r \rightarrow+\infty,
$$

moreover, the asymptotic relation (3) holds uniformly relative to $\varphi \in[-\pi, \pi),\left|\varphi-\theta_{j}\right| \geq \delta$, $0<\delta<1$.

Theorem 2. Let $\widetilde{v} \in L, f \in H_{0}\left(v, \Gamma_{m}^{\gamma}\right)$ and the asymptotic relation (3) holds with some function $G(\varphi)$ instead of the function $h_{f}(\varphi), G \in L^{1}[0,2 \pi]$. Then zeros of $f$ have $v$-density along c.r.r. $l_{\varphi}^{\gamma}$ for $f$, moreover, for all

$$
\alpha, \beta \in[-\pi, \pi) \backslash\left\{\bigcup_{j=1}^{m} \theta_{j}\right\}: \Delta^{\gamma}(\alpha, \beta)=\frac{1}{2 \pi}(G(\alpha)-G(\beta)+\Delta(\beta-\alpha)) .
$$

Remark 1. Under conditions of Theorem 1 if instead of (2) the following condition holds $(j=\overline{1, m})$

$$
n\left(r ; \theta_{j}\right)=\Delta_{j} v(r)+o(v(r)), r \rightarrow+\infty,
$$

then for $\varphi \in[-\pi, \pi), \varphi \neq \theta_{j}$,

$$
F(z)=\Delta v(r)+o(v(r)), r \rightarrow+\infty .
$$

3. Additional results. Without loss of generality, for $v \in L, f \in H_{0}(v)$ we assume that $v(r)=0$ as $0 \leq r \leq 1, f(0)=1$. To prove Theorems $1-2$ we will use following results, which we formulate as lemmas.

Lemma 1. Let $v \in L, w \in l_{-\pi}^{\gamma}, \beta(t)-\gamma(t) \rightarrow 0$ as $t \rightarrow+\infty$ and let $\alpha(t)$ be piecewise continuous function on $[1,+\infty), \alpha(t) \rightarrow 0$ as $t \rightarrow+\infty$. Then for $z=r e^{i(\varphi+\beta(r))},-\pi<\varphi<\pi$, we have

$$
\begin{gathered}
J_{1}=z \int_{l_{-\pi}^{\gamma}} \frac{\alpha(|w|) v(|w|)}{(w-z)^{2}} d w=o(v(r)), r \rightarrow+\infty \\
J_{2}=\int_{l_{-\pi}^{\gamma}(1, r)} \frac{\alpha(|w|) v(|w|)}{w-z} d w=o(v(r)), r \rightarrow+\infty \\
J_{3}=z \int_{l_{-\pi}^{\gamma}(r,+\infty)} \frac{\alpha(|w|) v(|w|)}{w(w-z)} d w=o(v(r)), r \rightarrow+\infty,
\end{gathered}
$$

moreover, the above relations hold uniformly relative to $\varphi \in[-\pi+\delta, \pi-\delta], 0<\delta<1$. 
Proof. Let $\varepsilon>0$ be an arbitrary fixed number, $K_{1}, K_{2}, \ldots$ be positive constants. We set $\eta=e^{-\delta /(4|c|)}$ with $c=\lim _{t \rightarrow+\infty}\left(t \gamma^{\prime}(t)\right)$. If $c=0$, then we assume $\eta=1 / 2$. Since $d w=$ $\left(1+i t \gamma^{\prime}(t)\right) e^{i(-\pi+\gamma(t))} d t$, we get

$$
\begin{gathered}
\left|J_{1}\right| \leq r \int_{1}^{+\infty} \frac{|\alpha(t)| v(t)}{|w-z|^{2}}\left|1+i t \gamma^{\prime}(t)\right| d t \leq K_{1} r\left(\int_{1}^{\eta r}+\int_{\eta r}^{r / \eta}+\int_{r / \eta}^{+\infty}\right) \frac{|\alpha(t)| v(t)}{|w-z|^{2}} d t= \\
=J_{1,1}+J_{1,2}+J_{1,3} .
\end{gathered}
$$

Since

$$
|w-z| \geq|| w|-| z||=|t-r|, \int_{1}^{\tau}|\alpha(t)| d t=o(\tau), \tau \rightarrow+\infty
$$

we have

$$
\left|J_{1,1}\right| \leq K_{1} r \int_{1}^{\eta r} \frac{|\alpha(t)| v(t)}{(r-\eta r)^{2}} d t \leq K_{2} \frac{v(r)}{r} \int_{1}^{\eta r}|\alpha(t)| d t<\frac{\varepsilon}{3} v(r), \quad r \geq r_{1} .
$$

Similarly,

$$
\left|J_{1,3}\right| \leq K_{1} r \int_{r / \eta}^{+\infty} \frac{|\alpha(t)| v(t)}{t^{2}\left(1-\frac{r}{t}\right)^{2}} d t \leq K_{2} r \int_{r / \eta}^{+\infty} \frac{|\alpha(t)| v(t)}{t^{2}} d t<\frac{\varepsilon}{3} v(r), \quad r \geq r_{2},
$$

because

$$
\int_{\tau}^{+\infty} \frac{|\alpha(t)| v(t)}{t^{2}} d t=o\left(\frac{v(\tau)}{\tau}\right), \tau \rightarrow+\infty .
$$

Taking into account [2, p. 605] that for arbitrary $b>0$

$$
\lim _{t \rightarrow+\infty}(\gamma(b t)-\gamma(t))=c \ln b
$$

for $t \in[\eta r, r / \eta]$ we obtain

$$
\widetilde{\varphi}=\varphi+\beta(r)-\gamma(t)=\varphi+(\beta(r)-\gamma(r))+(\gamma(r)-\gamma(t)) \rightarrow \varphi+c \ln b^{*}
$$

as $r \rightarrow+\infty, b^{*} \in[\eta, 1 / \eta]$. If $\varphi \in[-\pi+\delta, \pi-\delta]$ then for $r \geq r_{3}$

$$
\begin{gathered}
\widetilde{\varphi} \geq\left(\varphi+c \ln b^{*}\right)-\frac{\delta}{2} \geq-\pi+\frac{\delta}{2}-\left|c \ln b^{*}\right| \geq-\pi+\frac{\delta}{2}-|c| \ln e^{\delta /(4|c|)}=-\pi+\frac{\delta}{4}, \\
\widetilde{\varphi} \leq\left(\varphi+c \ln b^{*}\right)+\frac{\delta}{2} \leq \pi-\frac{\delta}{2}+|c| \ln e^{\delta /(4|c|)}=\pi-\frac{\delta}{4},
\end{gathered}
$$

and therefore $|\widetilde{\varphi}| \leq \pi-\frac{\delta}{4}$. Owing to inequality (see for example [4, p. 92]) $\left|t+r e^{i \theta}\right| \geq$ $(t+r) \sin \left(\delta_{1} / 2\right)$ as $|\theta| \leq \pi-\delta_{1}, 0<\delta_{1}<1$, we obtain

$$
|w-z|=\left|t e^{i(-\pi+\gamma(t))}-r e^{i(\varphi+\beta(r))}\right|=\left|t+r e^{i \widetilde{\varphi}}\right| \geq(t+r) \sin \frac{\delta}{8} .
$$

Let us put $\alpha^{*}(r)=\sup \{|\alpha(t)|: \eta r \leq t \leq r / \eta\}$. Since $\alpha^{*}(r) \rightarrow 0$ as $r \rightarrow+\infty$ we have for $r \geq r_{4}$

$$
\left|J_{1,2}\right| \leq K_{1} r \int_{\eta r}^{r / \eta} \frac{|\alpha(t)| v(t) d t}{(t+r)^{2} \sin ^{2}(\delta / 8)} \leq K_{2} \alpha^{*}(r) v(r / \eta) r \int_{\eta r}^{r / \eta} t^{-2} d t \leq K_{3} \alpha^{*}(r) v(r)<\frac{\varepsilon}{3} v(r) .
$$

Using the estimates obtained for $J_{1, k}, k=1,2,3$, we deduce that $\left|J_{1}\right|<\varepsilon v(r)$ as $r \geq$ $r_{5}=\max \left\{r_{j}: 1 \leq j \leq 4\right\}, \varphi \in[-\pi+\delta / 8, \pi-\delta / 8]$, proving (5). The relations (6), (7) can be proved similarly. Lemma 1 is proved. 
Let $v \in L, t \gamma^{\prime}(t)=c+\widetilde{\psi}_{v}(t)$ with $\widetilde{\psi}_{v}(t)=\alpha(t) \psi(t ; v), \alpha(t) \rightarrow 0$ as $t \rightarrow+\infty$ (see (1)), $k \in \mathbb{N} \cup\{0\}$. We set

$$
A_{k}(\tau, v)=\int_{1}^{\tau} v(t) t^{k} e^{i(k+1) \gamma(t)} d t, \quad B_{k}(\tau, v)=\int_{\tau}^{+\infty} v(t) t^{-k-2} e^{-i(k+1) \gamma(t)} d t .
$$

Lemma 2. If $\widetilde{v} \in L$, then

$$
\begin{gathered}
A_{k}(\tau, v)=\frac{1}{1+i c}\left(\frac{v(\tau) \tau^{k+1}}{k+1} e^{i(k+1) \gamma(\tau)}-\frac{1}{k+1} A_{k}(\tau, \widetilde{v})-i A_{k}\left(\tau, v \widetilde{\psi}_{v}\right)\right), \\
B_{k}(\tau, v)=\frac{1}{1+i c}\left(\frac{v(\tau) \tau^{-k-1}}{k+1} e^{-i(k+1) \gamma(\tau)}+\frac{1}{k+1} B_{k}(\tau, \widetilde{v})-i B_{k}\left(\tau, v \widetilde{\psi}_{v}\right)\right) .
\end{gathered}
$$

Proof. Integrating by parts we obtain

$$
\begin{gathered}
A_{k}(\tau, v)=\left.\frac{v(t) t^{k+1}}{k+1} e^{i(k+1) \gamma(t)}\right|_{1} ^{\tau}-\frac{1}{k+1} \int_{1}^{\tau} t^{k+1} e^{i(k+1) \gamma(t)}\left(v^{\prime}(t)+i(k+1) \gamma^{\prime}(t) v(t)\right) d t= \\
=\frac{v(\tau) \tau^{k+1} e^{i(k+1) \gamma(\tau)}}{k+1}-\frac{1}{k+1} \int_{1}^{\tau}\left(t v^{\prime}(t)\right) t^{k} e^{i(k+1) \gamma(t)} d t-i \int_{1}^{\tau} v(t)\left(t \gamma^{\prime}(t)\right) t^{k} e^{i(k+1) \gamma(t)} d t= \\
=\frac{v(\tau) \tau^{k+1} e^{i(k+1) \gamma(\tau)}}{k+1}-\frac{1}{k+1} A_{k}(\tau, \widetilde{v})-i c A_{k}(\tau, v)-i A_{k}\left(\tau, v \widetilde{\psi}_{v}\right) .
\end{gathered}
$$

Moving the expression $\left(-i c A_{k}(\tau, v)\right)$ to the left-hand side of the equality and dividing both sides into the multiplier $(1+i c)$ we find $(8)$.

The relation (9) can be obtained similarly. Thus, Lemma 2 is proved.

Lemma 3. Let $\widetilde{v} \in L, w \in l_{-\pi}^{\gamma}, \beta(r)-\gamma(r) \rightarrow 0$ as $r \rightarrow+\infty, 0<\delta<1$. Then for $z=r e^{i(\varphi+\beta(r))}$ the following relations hold uniformly relative to $\varphi \in[-\pi+\delta, \pi-\delta](r \rightarrow+\infty)$

$$
\begin{gathered}
I_{1}=z \int_{l_{-\pi}^{\gamma}(1, r)} \frac{v(|w|)}{(z-w)^{2}} d w=(1+i c) \lim _{\varepsilon \rightarrow 0+} \sum_{k=0}^{+\infty} \frac{(-1)^{k+1}(k+1)}{z^{k+1}} A_{k}((1-\varepsilon) r, v)+o(\widetilde{v}(r)) \\
I_{2}=z \int_{l_{-\pi}^{\gamma}(r,+\infty)} \frac{v(|w|)}{(z-w)^{2}} d w=(1+i c) \lim _{\varepsilon \rightarrow 0+} \sum_{k=0}^{+\infty} \frac{(-1)^{k+1}(k+1)}{z^{-k-1}} B_{k}((1+\varepsilon) r, v)+o(\widetilde{v}(r)) ; \\
I_{3}=\int_{l_{-\pi}^{\gamma}(1, r)} \frac{v(|w|) d w}{z-w}=(1+i c) \lim _{\varepsilon \rightarrow 0+} \sum_{k=0}^{+\infty} \frac{(-1)^{k+1}}{z^{k+1}} A_{k}((1-\varepsilon) r, v)+o(\widetilde{v}(r)) ; \\
I_{4}=z \int_{l_{-\pi}^{\gamma}(r,+\infty)} \frac{v(|w|) d w}{w(z-w)}=(1+i c) \lim _{\varepsilon \rightarrow 0} \sum_{k=0}^{+\infty} \frac{(-1)^{k+1}}{z^{-k-1}} B_{k}((1+\varepsilon) r, v)+o(\widetilde{v}(r)) .
\end{gathered}
$$

Proof. We have $w=t e^{i(-\pi+\gamma(t))}, d w=\left(1+i t \gamma^{\prime}(t)\right) e^{i(-\pi+\gamma(t))} d t=\left(1+i c+i \widetilde{\psi}_{v}(t)\right) e^{i(-\pi+\gamma(t))} d t$ and so that owing (5)

$$
I_{1}=\frac{1}{z} \int_{1}^{r} \frac{v(t)\left(1+i c+i \widetilde{\psi}_{v}(t)\right) e^{i(-\pi+\gamma(t))}}{\left(1-\frac{w}{z}\right)^{2}} d t=
$$




$$
\begin{gathered}
=(1+i c) \lim _{\varepsilon \rightarrow 0+} \int_{1}^{(1-\varepsilon) r}\left(\frac{v(t) e^{i(-\pi+\gamma(t))}}{z} \sum_{k=0}^{+\infty}(k+1)\left(\frac{w}{z}\right)^{k}\right) d t+ \\
+i z \int_{l_{-\pi}^{\gamma}(1, r)} \frac{\left(\alpha(t) /\left(1+i t \gamma^{\prime}(t)\right)\right) \widetilde{v}(t)}{(z-w)^{2}} d w= \\
=(1+i c) \lim _{\varepsilon \rightarrow 0+} \sum_{k=0}^{+\infty} \frac{(-1)^{k+1}(k+1)}{z^{k+1}} A_{k}((1-\varepsilon) r, v)+o(\widetilde{v}(r)), r \rightarrow+\infty .
\end{gathered}
$$

The relations (11)-(13) can be found similarly. Lemma 3 is proved.

The set $E \subset[0,+\infty)$ is called an $E_{0}$-set if mes $(E \cap[0, r])=o(r), r \rightarrow+\infty$. By Lemmas 4 and 5 from [8] we obtain the following proposition.

Lemma 4. Let $v \in L, 0<\delta<1, \theta \in[-\pi, \pi), f \in H_{0}(v)$. Then there exists $E_{0}$-set $E$ such that

$$
r \int_{\theta-\delta}^{\theta+\delta}\left|\frac{f^{\prime}\left(r e^{i \varphi}\right)}{f\left(r e^{i \varphi}\right)}\right| d \varphi=O(v(r))\left(\delta+\delta \ln \left(1+\frac{1}{\delta}\right)\right), r \rightarrow+\infty, r \notin E .
$$

\section{Proof of main results.}

Proof of Theorem 1. At first we assume that zeros of $f \in H_{0}(v)$ are located on c.r.r. $l_{-\pi}^{\gamma}$ for $f$. If $\left(a_{n}\right)_{n=1}^{+\infty}$ is a sequence of zeros of $f, 0<\left|a_{1}\right| \leq\left|a_{2}\right| \leq \ldots \leq\left|a_{n}\right| \leq \ldots \rightarrow+\infty$, then

$$
f(z)=\prod_{n=1}^{+\infty}\left(1-\frac{z}{a_{n}}\right), \quad \ln f(z)=\sum_{n=1}^{+\infty} \ln \left(1-\frac{z}{a_{n}}\right)
$$

where $\ln \left(1-\frac{z}{a_{n}}\right)$ is a single-valued branch in $\left(\mathbb{C} \backslash l_{-\pi}^{\gamma}\right)$ of the multivalued function $\operatorname{Ln}\left(1-\frac{z}{a_{n}}\right)$ such that $\left.\ln \left(1-\frac{z}{a_{n}}\right)\right|_{z=0}=0$. For $z=r e^{i(\varphi+\beta(r))},-\pi<\varphi<\pi$, we have (see (10),(11))

$$
\begin{gathered}
F(z)=z \frac{f^{\prime}(z)}{f(z)}=z \sum_{n=1}^{+\infty} \frac{1}{z-a_{n}}=z \int_{l_{-\pi}^{\gamma}} \frac{1}{z-w} d n(|w|)=-z \int_{l_{-\pi}^{\gamma}} \frac{n(|w|) d w}{(z-w)^{2}}= \\
=-z \int_{l_{-\pi}^{\gamma}} \frac{n(|w|)-v(|w|)}{(z-w)^{2}} d w-z \int_{l_{-\pi}^{\gamma}(1, r)} \frac{v(|w|) d w}{(z-w)^{2}}-z \int_{l_{-\pi}^{\gamma}(r,+\infty)} \frac{v(|w|) d w}{(z-w)^{2}}=\widetilde{J}-I_{1}-I_{2} .
\end{gathered}
$$

Since $n(r)=v(r)+\varepsilon(r) \widetilde{v}(r), \varepsilon(r) \rightarrow 0$ as $r \rightarrow+\infty$, then owing to (5)

$$
\widetilde{J}=-z \int_{l_{-\pi}^{\gamma}} \frac{\varepsilon(|w|) \widetilde{v}(|w|)}{(z-w)^{2}} d w=o(\widetilde{v}(r)), r \rightarrow+\infty .
$$

From (10) and (8) we obtain

$$
\begin{aligned}
I_{1}= & \lim _{\varepsilon \rightarrow 0+} \sum_{k=0}^{+\infty} \frac{(-1)^{k+1}(k+1)}{z^{k+1}}\left(\frac{v((1-\varepsilon) r)(1-\varepsilon)^{k+1} r^{k+1}}{k+1} e^{i(k+1) \gamma((1-\varepsilon) r)}-\frac{1}{k+1} A_{k}((1-\varepsilon) r, \widetilde{v})-\right. \\
& \left.-i A_{k}\left((1-\varepsilon) r, v \widetilde{\psi}_{v}\right)\right)+o(\widetilde{v}(r))=-v(r) \lim _{\varepsilon \rightarrow 0+} \frac{(1-\varepsilon) e^{i(\gamma((1-\varepsilon) r)-\beta(r)-\varphi)}}{1+(1-\varepsilon) e^{i(\gamma((1-\varepsilon) r)-\beta(r)-\varphi)}}+
\end{aligned}
$$




$$
\begin{gathered}
+\lim _{\varepsilon \rightarrow 0+} \sum_{k=0}^{+\infty} \frac{(-1)^{k}}{z^{k+1}} A_{k}((1-\varepsilon) r, \widetilde{v})+i \lim _{\varepsilon \rightarrow 0+} \sum_{k=0}^{+\infty} \frac{(-1)^{k}(k+1)}{z^{k+1}} A_{k}\left((1-\varepsilon) r, v \widetilde{\psi}_{v}\right)+o(\widetilde{v}(r))= \\
=-v(r) \frac{e^{i(\gamma(r)-\beta(r)-\varphi)}}{1+e^{i(\gamma(r)-\beta(r)-\varphi)}}+\Sigma_{1}+i \Sigma_{2}+o(\widetilde{v}(r)), r \rightarrow+\infty .
\end{gathered}
$$

Further, from (10), (5)

$$
\Sigma_{2}=\frac{-z}{1+i c} \int_{l_{-\pi}^{\gamma}} \frac{\alpha(|w|) \widetilde{v}(|w|)}{(z-w)^{2}} d w=o(\widetilde{v}(r)), r \rightarrow+\infty,
$$

also, owing to (8), (12) and (6)

$$
\begin{gathered}
\Sigma_{1}=\frac{1}{1+i c} \lim _{\varepsilon \rightarrow 0+} \sum_{k=0}^{+\infty} \frac{(-1)^{k}}{z^{k+1}}\left(\frac{\widetilde{v}((1-\varepsilon) r)(1-\varepsilon)^{k+1} r^{k+1}}{k+1} e^{i(k+1) \gamma((1-\varepsilon) r)}-\frac{1}{k+1} A_{k}\left((1-\varepsilon) r, \widetilde{v}_{\mathrm{ln}}^{\prime}\right)-\right. \\
\left.-i A_{k}\left((1-\varepsilon) r, \widetilde{v} \widetilde{\psi}_{\widetilde{v}}\right)\right)=\frac{1}{1+i c} \widetilde{v}(r) \lim _{\varepsilon \rightarrow 0+} \sum_{k=0}^{+\infty} \frac{(-1)^{k}}{k+1}\left((1-\varepsilon) e^{i(\gamma((1-\varepsilon) r)-\beta(r)-\varphi)}\right)^{k+1}- \\
-\frac{1}{1+i c} \lim _{\varepsilon \rightarrow 0+} \sum_{k=0}^{+\infty} \frac{(-1)^{k}}{(k+1) z^{k+1}} A_{k}\left((1-\varepsilon) r, \widetilde{v}_{\mathrm{ln}}^{\prime}\right)+\frac{i}{(1+i c)^{2}} \int_{l_{-\pi}^{\gamma}(1, r)} \frac{\widetilde{\psi}_{\widetilde{v}}(t) \widetilde{v}(t)}{z-w} d w+o(\widetilde{v}(r))= \\
=\frac{\widetilde{v}(r)}{1+i c} \ln \left(1+e^{i(\gamma(r)-\beta(r)-\varphi)}\right)-\frac{1}{1+i c} \lim _{\varepsilon \rightarrow 0+} \Sigma_{\varepsilon}+o(\widetilde{v}(r)), r \rightarrow+\infty .
\end{gathered}
$$

Setting $\varphi_{1}(r)=\varphi+\beta(r)-\gamma(r)$,

$$
a_{k}^{*}(r, v)=\frac{1}{k+1} \int_{1}^{r} v(t) t^{k} d t
$$

by Lemma 3 from [7] we have

$$
\left|\Sigma_{\varepsilon}\right| \leq \sum_{k=0}^{+\infty} \frac{1}{(k+1) r^{k+1}} \int_{1}^{r} \widetilde{v}_{\mathrm{ln}}^{\prime}(t) t^{k} d t=\sum_{k=0}^{+\infty} \frac{a_{k}^{*}\left(r, \widetilde{v}_{\mathrm{ln}}^{\prime}\right)}{r^{k+1}}=o(\widetilde{v}(r)), r \rightarrow+\infty,
$$

and hence

$$
I_{1}=-\frac{v(r) e^{-i \varphi_{1}(r)}}{1+e^{-i \varphi_{1}(r)}}+\frac{\widetilde{v}(r)}{1+i c} \ln \left(1+e^{-i \varphi_{1}(r)}\right)+o(\widetilde{v}(r)), r \rightarrow+\infty .
$$

Similarly using (11), (5), (9), (13), (7) and Lemma 3 from [7] one can obtain

$$
I_{2}=-\frac{v(r)}{1+e^{-i \varphi_{1}(r)}}-\frac{\widetilde{v}(r)}{1+i c} \ln \left(1+e^{i \varphi_{1}(r)}\right)+o(\widetilde{v}(r)), r \rightarrow+\infty .
$$

From (14)-(17) we have for $z=r e^{i(\varphi+\beta(r))},-\pi<\varphi<\pi$,

$$
\begin{gathered}
F(z)=v(r)+\frac{1}{1+i c} \ln \frac{1+e^{i \varphi_{1}(r)}}{1+e^{-i \varphi_{1}(r)}} \widetilde{v}(r)+o(\widetilde{v}(r))=v(r)+\frac{1}{1+i c} i \varphi_{1}(r) \widetilde{v}(r)+o(\widetilde{v}(r))= \\
=v(r)+i(1+i c)^{-1} \varphi \widetilde{v}(r)+o(\widetilde{v}(r)), r \rightarrow+\infty .
\end{gathered}
$$


If zeros of $f \in H_{0}(v)$ lies on c.r.r. $l_{\theta}^{\gamma},-\pi<\theta<\pi$, for $f$ then by rotating the plane on the angle $(\pi+\theta)$ in a clockwise direction, that is by substituting the quantity $(\varphi-\theta-\pi)$ for $\varphi$ in (18) uniformly relative to $\varphi \in[\theta+\delta, \theta+2 \pi-\delta], 0<\delta<1$ the following asymptotic relation holds

$$
F\left(r e^{i(\varphi+\beta(r))}\right)=v(r)+i(1+i c)^{-1}(\varphi-\theta-\pi) \widetilde{v}(r)+o(\widetilde{v}(r)), r \rightarrow+\infty .
$$

Let $f$ satisfy the assumptions of Theorem 1, namely zeros of $f$ are located on $\Gamma_{m}^{\gamma}(f)$ and (2) holds. We represent $f$ by a product of the form $f=f_{1} \cdot \ldots \cdot f_{m}$, where $f_{j}$ is an entire function with zeros on c.r.r. $l_{\theta_{j}}^{\gamma}, j=\overline{1, m}$. Then for $z \in\left(\mathbb{C} \backslash \Gamma_{m}^{\gamma}\right)$ we have $\ln f(z)=$ $\ln f_{1}(z)+\ldots+\ln f_{m}(z)$ and using the last relation for $z=r e^{i(\varphi+\gamma(r))}, \varphi \neq \theta_{j}$ we obtain

$$
\begin{gathered}
F(z)=z \sum_{j=1}^{m} \frac{f_{j}^{\prime}(z)}{f_{j}(z)}=\Delta v(r)+\frac{i}{1+i c} \sum_{j=1}^{m} \Delta_{j} \widehat{h}_{j}(\theta) \widetilde{v}(r)+o(\widetilde{v}(r))= \\
=\Delta v(r)+\frac{i}{1+i c} h_{f}(\theta) \widetilde{v}(r)+o(\widetilde{v}(r)), r \rightarrow+\infty .
\end{gathered}
$$

This completes the proof of Theorem 1 .

Proof of Theorem 2. Let $\Omega=\left(a_{n}\right)_{n=1}^{+\infty}$ be a sequence of zeros of $f \in H_{0}\left(v, \Gamma_{m}^{\gamma}\right)$,

$$
\partial D^{\gamma}(r ; \alpha, \beta)=l_{\alpha}^{\gamma}(1, r) \cup C_{r}(\alpha, \beta) \cup\left(l_{\beta}^{\gamma}(1, r)\right)^{-1} \cup\left(C_{1}(\alpha, \beta)\right)^{-1}
$$

be the positive orientation of the curvilinear sector $D^{\gamma}(r ; \alpha, \beta)$ with $-\pi \leq \theta_{1}<\ldots<\theta_{k_{0}-1}<$ $\alpha<\theta_{k_{0}}<\ldots<\theta_{l_{0}}<\beta<\ldots<\theta_{m}<\pi, r \notin \Omega, C_{\tau}(\alpha, \beta)=\left\{z=\tau e^{i \varphi}, \alpha \leq \varphi \leq \beta\right\}$. Then

$$
\begin{gathered}
2 \pi i n^{\gamma}(r ; \alpha, \beta)=\int_{\partial D^{\gamma(r ; \alpha, \beta)} \frac{f^{\prime}(z)}{f(z)}} d z=\left(\int_{l_{\alpha}^{\gamma}(1, r)}+\int_{C_{r}(\alpha, \beta)}-\int_{l_{\beta}^{\gamma}(1, r)}-\int_{C_{1}(\alpha, \beta)}\right) \frac{f^{\prime}(z)}{f(z)} d z= \\
=A_{1}+A_{2}+A_{3}+A_{4} .
\end{gathered}
$$

By the conditions of Theorem 2 we obtain $\left(\widetilde{v}(t)=t v^{\prime}(t)\right)$

$$
\begin{gathered}
A_{1}=\int_{1}^{r} \frac{f^{\prime}\left(t e^{i(\alpha+\gamma(t))}\right)}{f\left(t e^{i(\alpha+\gamma(t))}\right)}\left(1+i t \gamma^{\prime}(t)\right) e^{i(\alpha+\gamma(t))} d t=(1+i c) \int_{1}^{r} \frac{F\left(t e^{i(\alpha+\gamma(t))}\right)}{t} d t+ \\
+i \int_{1}^{r} \frac{F\left(t e^{i(\alpha+\gamma(t))}\right) \widetilde{\psi}_{v}(t)}{t} d t=(1+i c) \Delta \int_{1}^{r} \frac{v(t)}{t} d t+i \int_{1}^{r}\left(G(\alpha) v^{\prime}(t)+\varepsilon_{1}(t) v^{\prime}(t)\right) d t+ \\
+i \Delta \int_{1}^{r} \varepsilon(t) v^{\prime}(t) d t-\frac{1}{1+i c} \int_{1}^{r} \frac{\varepsilon_{2}(t)}{t}(G(\alpha) \widetilde{v}(t)+o(\widetilde{v}(t))) d t= \\
=(1+i c) \Delta v_{1}(r)+i G(\alpha) v(r)+o(v(r)), r \rightarrow+\infty
\end{gathered}
$$

with

$$
v_{1}(r)=\int_{1}^{r} v(t) / t d t, \quad \varepsilon_{j}(t) \rightarrow 0
$$

as $t \rightarrow+\infty, j=1,2$. Similarly,

$$
A_{3}=-(1+i c) \Delta v_{1}(r)-i G(\beta) v(r)+o(v(r)), r \rightarrow+\infty .
$$


Let

$$
0<\delta<\min \left\{\frac{\theta_{k_{0}}-\alpha}{2}, \frac{\beta-\theta_{l_{0}}}{2}, \frac{\theta_{j+1}-\theta_{j}}{2}\right\}
$$

$j=\overline{k_{0}, l_{0}-1}$. Then taking into account (3) we have

$$
\begin{gathered}
A_{2}=i\left(\int_{\alpha}^{\theta_{k_{0}}-\delta}+\sum_{j=k_{0}}^{l_{0}-1} \int_{\theta_{j}+\delta}^{\theta_{j+1}-\delta}+\int_{\theta_{l_{0}}+\delta}^{\beta}\right) F\left(r e^{i(\varphi+\gamma(r))}\right) d \varphi+i \sum_{j=k_{0}}^{l_{0}} \int_{\theta_{j}-\delta}^{\theta_{j}+\delta} F\left(r e^{i(\varphi+\gamma(r))}\right) d \varphi= \\
=i \Delta v(r)\left(\left(\theta_{k_{0}}-\delta-\alpha\right)+\sum_{j=k_{0}}^{l_{0}-1}\left(\theta_{j+1}-\theta_{j}-2 \delta\right)+\left(\beta-\theta_{l_{0}}-\delta\right)\right)+i \Sigma_{\delta}+o(v(r))= \\
=i \Delta\left(\beta-\alpha-2 \delta\left(l_{0}-k_{0}+1\right)\right) v(r)+i \Sigma_{\delta}+o(v(r)), r \rightarrow+\infty
\end{gathered}
$$

with

$$
\Sigma_{\delta}=\sum_{j=k_{0}}^{l_{0}} \int_{\theta_{j}-\delta}^{\theta_{j}+\delta} F\left(r e^{i(\varphi+\gamma(r))}\right) d \varphi
$$

By Lemma 4 there exists an $E_{0}$-set $E_{j}$ such that

$$
\left|\int_{\theta_{j}-\delta}^{\theta_{j}+\delta} F\left(r e^{i(\varphi+\gamma(r))}\right) d \varphi\right|=O(v(r))\left(\delta+\delta \ln \left(1+\frac{1}{\delta}\right)\right), r \rightarrow+\infty, r \notin E_{j},
$$

and hence

$$
\left|\Sigma_{\delta}\right| \leq O(v(r))\left(\delta+\delta \ln \left(1+\frac{1}{\delta}\right)\right), r \rightarrow+\infty, r \notin E=\bigcup_{j=k_{0}}^{l_{0}} E_{j} .
$$

Sending $\delta$ to zero from (19)-(23) we obtain

$$
\frac{n^{\gamma}(r ; \alpha, \beta)}{v(r)}=\frac{1}{2 \pi}(G(\alpha)-G(\beta)+\Delta(\beta-\alpha))+o(1), r \rightarrow+\infty, r \notin E .
$$

Let us show that the equality (24) is fulfilled for $r \in[1,+\infty)$. Indeed, let us suppose that $r \in E$. From the definition of $E_{0}$-set it follows that there exist numbers $r^{\prime}, r^{\prime \prime}$ such that $\frac{r}{2}<r^{\prime}<r<r^{\prime \prime}<2 r, r^{\prime} \notin E, r^{\prime \prime} \notin E$. Since

$$
\frac{n^{\gamma}\left(r^{\prime} ; \alpha, \beta\right)}{v\left(r^{\prime}\right)} \frac{v\left(r^{\prime}\right)}{v(r)} \leq \frac{n^{\gamma}(r ; \alpha, \beta)}{v(r)} \leq \frac{n^{\gamma}\left(r^{\prime \prime} ; \alpha, \beta\right)}{v\left(r^{\prime \prime}\right)} \frac{v\left(r^{\prime \prime}\right)}{v(r)},
$$

$v\left(r^{\prime}\right) \sim v\left(r^{\prime \prime}\right) \sim v(r), r \rightarrow+\infty$, then from $(24)$

$$
\lim _{r \rightarrow+\infty} \frac{n^{\gamma}(r ; \alpha, \beta)}{v(r)}=\frac{1}{2 \pi}(G(\alpha)-G(\beta)+\Delta(\beta-\alpha))=\Delta^{\gamma}(\alpha, \beta) .
$$

This completes the proof of Theorem 2 .

Remark 2. If the conditions of Theorem 2 are fulfilled with

$$
G(\theta)=h_{f}(\theta), \quad \Delta=\sum_{j=1}^{m} \Delta_{j}
$$

then $\Delta^{\gamma}(\alpha, \beta)=\sum_{j=k_{0}}^{l_{0}} \Delta_{j}$, where $\alpha \in\left(\theta_{k_{0}-1}, \theta_{k_{0}}\right), \beta \in\left(\theta_{l_{0}}, \theta_{l_{0}+1}\right)$. 


\section{REFERENCES}

1. S.K. Balashov, On entire functions of completely regular growth along curves of regular rotation, Math. USSR Izv., 10 (1976), №2, 321-338. doi:10.1070/IM1976v010n02ABEH001691; translation of Izv. Akad. Nauk SSSR Ser. Mat., 40 (1976), №2, 338-354. (in Russian)

2. S.K. Balashov, On entire functions of finite order with zeros on curves of regular rotation, Math. USSR Izv., 7 (1973), №3, 601-627. doi:10.1070/IM1973v007n03ABEH001963; translation of Izv. Akad. Nauk SSSR Ser. Mat., 37 (1973), №3, 603-629. (in Russian)

3. A.A. Goldberg, N.E. Korenkov, Asymptotic behavior of logarithmic derivative of entire function of completely regular growth, Sib. Math. J., 21 (1980), №3, 363-375. doi:10.1007/BF00968180; translation of Sibirsk. Mat. Zh., 21 (1980), №3, 63-79. (in Russian)

4. A.A. Goldberg, I.V. Ostrovskii, Value distribution of meromorphic functions, Translation of Mathematical Monographs, V.236, AMS, 2008.

5. A.A. Goldberg, N.N. Strochik, Asymptotic behavior of meromorphic functions of completely regular growth and of their logarithmic derivatives, Sib. Math. J., 26 (1985), №6, 802-809. doi:10.1007/BF00969100; translation of Sibirsk. Mat. Zh., 26 (1985), №6, 29-38. (in Russian)

6. A.A. Goldberg, N.V. Zabolotskii, Concentration index of a subharmonic function of zeroth order, Math. Notes, 34 (1983), №2, 596-601. doi:10.1007/BF01141775; translation of Mat. Zametki, 34 (1983), №2, 227-236. (in Russian)

7. M.V. Zabolotskii, Valiron-type and Valiron-Titchmarsh-type theorems for entire functions of order zero, Ukrainian Math. J., 48 (1996), №3, 354-366. doi:10.1007/BF02378526; translation of Ukrain. Mat. Zh., 48 (1996), №3, 315-325. (in Ukrainian)

8. N.V. Zabolotskii, Strongly regular growth of entire functions of order zero, Math. Notes, 63 (1998), №2, 172-182. doi:10.1007/BF02308756; translation of Mat. Zametki, 63 (1998), №2, $196-208$. doi:10.4213/mzm1266 (in Russian)

9. M.V. Zabolotskyi, Yu.V. Basyuk, S.I. Tarasyuk, Entire functions of order zero with zeros on a logarithmic spiral, Ukrainian Math. J., 70 (2018), №7, 1063-1074. doi:10.1007/s11253-018-1552-6; translation of Ukrain. Mat. Zh., 70 (2018), №7, 923-932. (in Ukrainian)

10. M.V. Zabolotskyi, M.R. Mostova, Sufficient conditions for the existence of the $v$-density of zeros for an entire function of order zero, Ukrainian Math. J., 68 (2016), №4, 570-582. doi:10.1007/s11253-016-12421; translation of Ukrain. Mat. Zh., 68 (2016), №4, 506-516. (in Ukrainian)

11. M.V. Zabolotskyj, M.R. Mostova, Asymptotic behavior of the logarithmic derivative of entire functions of zero order, Carpathian Math. Publ., 6 (2014), №2, 237-241. doi:10.15330/cmp.6.2.237-241 (in Ukrainian)

Ivan Franko National University of Lviv

Lviv, Ukraine

mykola.zabolotskyy@lnu.edu.ua, yuliya.basyuk.92@gmail.com

Ivan Bobersky Lviv State University of Physical Culture

Lviv, Ukraine

mariana.mostova@gmail.com 